\title{
Laboratory measurements of the sedimentation velocity of hexagonal planar ice crystals
}

Rodrigo E. Bürgesser and Nesvit E. Castellano

\author{
Group of Atmospheric Physics, FaMAF, Universidad Nacional de Córdoba, IFEG- \\ CONICET, Córdoba, Argentina. \\ Corresponding author: Rodrigo Bürgesser, burgesse@famaf.unc.edu.ar. FAMAF, \\ Ciudad Universitaria, 5000 Córdoba, Argentina.
}

\begin{abstract}
New experimental measurements of the falling velocity, size and orientation of individual hexagonal plate-like ice crystals are reported. The measurements were conducted at three different temperatures: $-13,-16$ and $-20^{\circ} \mathrm{C}$. The diameter of the ice crystals measured in the experiments were between $50 \mu \mathrm{m}$ and $250 \mu \mathrm{m}$, a size range which is in agreement with the size found in natural clouds. In this range, ice crystals show a random orientation during free fall and a falling velocity which increases with size. Results show that the fall velocity is insensitive to the temperature at which the ice crystals grow for the temperatures used on this study. An empirical power-law between the Best and Reynolds number is presented using the capacitance as characteristic length and an estimation of the ice crystal mass. Despite the dispersion of the experimental data, the Best-Reynolds relationship found seems to be similar to the relationship for falling spheres in Stokes flow using the capacitance as the hydrodynamic radius. The fall velocity of hexagonal and columnar ice crystals were
\end{abstract}

This article has been accepted for publication and undergone full peer review but has not been through the copyediting, typesetting, pagination and proofreading process, which may lead to differences between this version and the Version of Record. Please cite this article as doi: $10.1002 /$ qj.3321 
compared. The columnar ice crystals show a velocity larger than that of hexagonal ice crystals with the same value of capacitance. However, both crystalline habits show an unique empirical Be-Re relationship.

Keywords: Fall velocity; Hexagonal planar ice crystals; Experimental data.

\section{Introduction}

Knowledge of the sedimentation velocity of ice crystals is necessary for the study and understanding of several microphysical processes in clouds. Furthermore, ice crystals have a relevant impact on the Earth's climate through their role in the radiative budget between the Earth and the Sun. Different attempts have been made to study and parameterize the sedimentation velocity using theoretical calculations, tank models and experimental measurements. However, the sedimentation process of ice crystals is still poorly characterized.

Given the non-spherical shape of ice crystals, theoretical calculations are hard to perform. A common approach is to determine a power-law relationship between Best number (Be) and Reynolds number (Re). Heymsfield (1972) calculated the terminal velocity of ice crystals with different habits using the drag coefficient, aspect ratio and density of the ice particles. Heymsfield and Kajikawa (1987), based on measurements of planar crystals and graupel reported by Kajikawa $(1972,1975)$, calculated the terminal velocity of those ice particles using a simple expression between Be and Re numbers for Re numbers higher than 5. Mitchell (1996) derived a theoretical relationship between Be and Re numbers for all ice particle types and found four power-law expressions for Be-Re over different ranges of Be. Khvorostyanov and Curry (2002) reported a continuous representation of the fall velocity of liquid and crystalline cloud particles in 
terms of $\mathrm{Be}$ and $\mathrm{Re}$ numbers over the full range of particle sizes observed in the atmosphere. Mitchell and Heymsfield (2005) and Khvorostyanov and Curry (2005) found a refined formulation of the Be-Re representation provided by Khvorostyanov and Curry (2002). However, these formulations are successful in predicting terminal velocity only for large Reynolds numbers $(\operatorname{Re}>>1)$. Meanwhile, for low Reynolds number, the theoretical formulation seems to overestimate the fall speed (Westbrook, 2008; Heymsfield and Westbrook, 2010).

The main experimental data available on ice crystal fall speeds are from the 70's decade (Kajikawa, 1973; Jayaweera and Ryan, 1972; Michaeli, 1977). These experimental studies reported the fall speed, size and mass of ice crystals of both main habits, plate- and column-like. These experimental studies used streak photographs of the falling ice crystals to determine their terminal velocity. The size and mass of the falling ice crystals were measured from replica samples taken during the experiments. Recently, Bürgesser et al. (2016) reported new experimental data of the fall velocity for columnar ice crystals. The measurements were performed using a high speed camera with an optical arrangement that allows measurement of the size and fall velocity of single ice crystals from the recorded data. These researchers found a linear relationship between the velocity and the capacitance (Westbrook et al., 2008) of columnar ice crystals with different aspect ratios. Using new technology, Bürgesser et al. (2016) were able to determine the fall speed, size and falling orientation of ice crystals with higher precision than in previous studies.

Due to the existence of little experimental data of the fall velocity of plate-like ice crystals and the dispersion observed in these data, together with the reported overestimation of the velocity calculated by theoretical models, the aim of this study is to perform experimental measurements of the fall speed, size and falling orientation of 
hexagonal planar ice crystals in a size range representative of natural ice crystals. The use of new technology allows to record each ice crystal individually and to measure its size and falling orientation. These new measurements expand the results reported by previous researchers and provide new information on the sedimentation process of ice crystals.

\section{Experimental device}

The measurements were conducted using the same experimental device and method described in Bürgesser et al. (2016), which is shown in Figure 1. The measurements were conducted at three different temperatures: $-13,-16$ and $-20^{\circ} \mathrm{C}$. These temperatures were chosen since they correspond to ice crystals with hexagonal plate-like basic habit (e.g. Bailey and Hallett, 2009).

A supercooled cloud of water droplets was generated by vapour condensation on natural cloud condensation nuclei. The ice crystals were generated in this supercooled cloud by cooling a local volume of the droplet cloud with a rapid expansion of air compressed inside a syringe (Castellano et al. 2014; Bürgesser et al. 2016).

After seeding, an ice crystal concentration between 200 and $500 \mathrm{l}^{-1}$ was estimated. These ice crystals grew by vapour deposition until they reached a size large enough to fall into the acrylic sedimentation tube, where the ice crystals were recorded with a high speed camera. From the recorded data, size, fall velocity and falling orientation of well-defined hexagonal plate ice crystals were measured.

The length of the sedimentation tube is large enough to ensure that ice crystals reach their terminal velocity at the position of the camera. To suppress the evaporation of the ice crystals during their fall in the sedimentation tube, a small amount of ice was placed at the bottom of the tube. Turbulence and thermal convection were avoided by 
keeping the bottom of the sedimentation tube a few degrees colder than the top of the tube.

Figure 2 (Left panel) shows the real size of an hexagonal plate-like ice crystal with dimensions, $\mathrm{D}$ and $\mathrm{L}$, which are the ice crystal diameter and thickness, respectively. The right panel of Figure 2 shows the projection of the ice crystal over the focal plane of the camera where the parameters measured in the experiments are indicated: the larger (D) and the shorter (d) dimensions of the projection of the ice crystals over the focal plane of the camera, and the angle between D and the vertical direction $(\alpha)$. As can be observed, due to the symmetry of the plate ice crystals, the larger dimension of the projected ice crystal is the diameter of the hexagonal plate.

To determine the falling and horizontal velocities, the position and time of each ice crystal were fit with a linear function. At least five points (position and time) were required to perform a fit. All linear fits showed $R^{2}>0.999$ which ensured that the ice crystals had reached their terminal velocity, and since the horizontal velocity is at least one order of magnitude smaller than the fall velocity, the free fall conditions was also assured.

\section{Results and Discussion}

Figure 3 shows the size distribution of the hexagonal plate crystals measured for the three temperatures used in this study. The mean and median ice-crystal sizes are given by the square and the horizontal line inside the boxes, respectively. The boxes indicate the standard deviation, and the small dashes the minimum and maximum sizes. Sizes range from $50 \mu \mathrm{m}$ to $250 \mu \mathrm{m}$, with median sizes of $139 \mu \mathrm{m}$ for $-13^{\circ} \mathrm{C}, 161 \mu \mathrm{m}$ for $-16^{\circ} \mathrm{C}$ and $98 \mu \mathrm{m}$ for $-20^{\circ} \mathrm{C}$. The sizes of the ice crystals are in agreement with those measured in natural clouds (Ono, 1970; Auer and Veal, 1970; Um et al. 2014). 
Figure 4 shows the measured fall velocity as a function of $\mathrm{D}$ for temperatures of $-13^{\circ} \mathrm{C}$ (black circles), $-16^{\circ} \mathrm{C}$ (black triangles) and $-20^{\circ} \mathrm{C}$ (black stars). The data dispersion observed for the terminal velocity of crystals of the same size seems to be due to the variations in crystal mass (Heymsfield, 1972) and the different crosssectional area to the flow presented by the ice crystals during their fall. For instance, Kajikawa (1973) melted the sampled ice crystals to measure their mass and reported the equivalent droplet diameter of the ice crystals as a function of their diameters (Figure 4 in Kajikawa, 1973). These results showed a large variability of the equivalent droplet diameter for a fixed ice crystal size, which represents a large variability of the ice crystal mass. On the other hand, Michaeli (1977) reported a dispersion of $15 \%$ on the mean ice crystal mass.

Despite the data dispersion observed in Figure 4, ice crystals show a tendency to increase velocity with size. Furthermore, the fall velocity does not show a dependence on temperature, in agreement with the result reported by Heymsfield (1972). The data reported by Kajikawa (1973) (open squares) and Michaeli (1977) (open circles) is also displayed on Figure 4. As can be observed, the data reported in this study are indistinguishable from those reported by Michaeli (1977). However, the velocities measured by Kajikawa (1973) are slightly higher than those reported here. Westbrook (2008) observed that, at least for the smaller ice crystals, Kajikawa (1973) underestimated the diameter of the ice crystals, which supports the present results.

Figure 5 (Upper panel) shows the fall velocity as a function of $\mathrm{D}$ for all temperatures measured. Open circles represent ice crystals with horizontal falling orientation $\left(\alpha=90^{\circ}\right)$ and black circles represent ice crystals with random falling orientation $\left(\alpha \neq 90^{\circ}\right)$ except for the horizontal one. As can be observed, there is no discernible trend on the falling velocity of the ice crystals with horizontal falling 
orientation. The solid line represents the best linear fit using a zero y-intercept. The slope of the fit between the fall velocity and D is $(0.031 \pm 0.001) \mathrm{cm} \mathrm{s}^{-1} \mu \mathrm{m}^{-1}$ with $R^{2}=0.872$. Figure 5 (Lower panel) shows the fall velocity as a function of $\mathrm{C}$ for all temperatures measured. The use of the capacitance (C) as a characteristic length for ice crystals was proposed by Westbrook (2008). This proposal was used by Bürgesser et al. (2016) who found a better representation of the fall velocity of columnar ice crystals using $\mathrm{C}$ as a characteristic length. Given that the length (L) of the ice crystals was not measured and its value is necessary to determine $\mathrm{C}$, this parameter was calculated using the relationship proposed by Auer and Veal (1970). Other parameterizations of L (Davis, 1974; Ono, 1970) were used to calculate $\mathrm{C}$ but the different values of $\mathrm{C}$ obtained did not show significant differences. However, using $\mathrm{C}$ as a characteristic dimension does not show an improvement in data representation. The best linear fit between the fall velocity and $\mathrm{C}$ was calculated using a zero y-intercept and the slope obtained was $(0.091 \pm 0.003) \mathrm{cm} \mathrm{s}^{-1} \mu \mathrm{m}^{-1}$ with $R^{2}=0.87568$.

The use of $\mathrm{C}$ as a characteristic length allows to compare the fall velocity of ice crystals with different habits. The broken line in Figure 5 (Lower panel) represents the linear relationship between the fall velocity and $\mathrm{C}$ for columnar ice crystals reported by Bürgesser et al. (2016). As can be observed, the columnar ice crystals show a trend to present larger fall velocity than hexagonal ice plates for the same value of $\mathrm{C}$.

The use of a linear fit between $\mathrm{D}$ or $\mathrm{C}$ and the fall velocity seems not to be the best approach given the data dispersion observed in Figure 5. Therefore, a more conventional approach using $\mathrm{Be}$ and Re numbers is presented. The Be and Re numbers were computed using the following definitions,

$$
B e=\frac{2 m g(2 C)^{2} \rho_{a i r}}{A \eta^{2}}
$$


$R e=\frac{(2 C) \rho_{a i r} V}{\eta}$

where $g$ is the acceleration due to gravity, $\rho_{\text {air }}$ is the density of the air, $\eta$ is the dynamic viscosity of the air, $A$ is the cross-sectional area which the particle presents to the flow, $m$ is the mass of the ice particle and $V$ is the fall velocity of the particle. Based on the proposal of Westbrook (2008) and the results reported by Bürgesser et al. (2016), C was used as the characteristic dimension of the ice crystals in the Be and Re definitions (Equations 1 and 2).

Given that the mass $m$ of the ice particles was not measured, it was estimated using geometry and by using different parameterizations. To estimate the mass using geometry, the length (L) of the ice crystals was calculated using the relationships proposed by different researchers (Auer and Veal, 1970; Davis, 1974; Ono, 1970) and the ice densities reported by Ryan et al. (1976). On the other hand, ice crystal masses were also estimated using different relationships between the mass and the diameter of ice crystals reported by several researchers (e.g. Mitchell, 1996; Schmitt and Heymsfield, 2009). Furthermore, the ice crystal mass was estimated using the data reported by Kajikawa (1973). However, the estimated values of the ice crystal mass using the different approaches show a large discrepancy with differences of up to one order of magnitude for the larger ice crystals. Therefore, in order to compute the Be number, the ice crystal mass was estimated using the mean value of all the parameterizations. For each ice crystal, an error was assigned to the Be number which takes this mass dispersion into account.

Figure 6 shows the Be-Re relationships measured for the ice crystals as well as the empirical power-law found. The data dispersion observed in Figure 6 is due to the 
mass variability of ice crystals, which is represented by the error assigned to the Be number. As was pointed out by Heymsfield (1972) and Heymsfield and Westbrook (2010), the ice crystal mass seems to be the primary driver for the sedimentation process. Open circles, in Figure 6, represent ice crystals with horizontal falling orientation $\left(\alpha=90^{\circ}\right)$, which show larger values on the Be number than ice crystals with different orientation and the same Re number. This behavior is due to the fact that these ice crystals present the minimum cross-sectional area to the flow.

The Be-Re analysis was performed for the different temperatures but the fitting parameters of the power-law did not show significant difference. Therefore, the Be-Re relationship is based on all the data measured and has the following form,

$B e=A R e^{\beta}$

where $\mathrm{A}=(42 \pm 5)$ and $\beta=(0.84 \pm 0.08)$, with a $\mathrm{R}^{2}$ value of 0.43644 . The power-law found is valid for Reynolds number between 0.1 and 1 and for hexagonal plate-like ice crystals.

The Best and Reynolds numbers for the data reported by Kajikawa (1973) and Michaeli (1977) for hexagonal ice plates is also plotted in Figure 6. The Be and Re values for Michaeli (1977) data were calculated using the size, mass and velocity reported by this researcher but using $\mathrm{C}$ as the characteristic length. Given that Kajikawa (1973) did not explicitly report the mass and length of the measured ice crystals, the Be number of his data were obtained using the mean mass value. The data reported by Michelli (1977) show an excellent agreement with the present measurements and with the power law found, while the data reported by Kajikawa (1973) show a good agreement with the present data for Re higher than 0.1 . For Re lower than 0.1 , the data 
of Kajikawa (1973) seem to underestimate the values of the Best number. These data correspond to the smaller ice crystals measured by this researcher which, according to Westbrook (2008), seem to be underestimated. However, the data reported here is only valid for Re values between 0.1 and 1 , and no further comparison is possible.

The Be-Re relationship found is similar to the Stokes solution for spheres of radius $\mathrm{C}$ based on viscous flow $(R e<<1)$. Given the data dispersion, this solution is also a good parameterization of the present data. Westbrook (2008), based on dimensional analysis, proposed that non-spherical ice particles falling in random orientation should follows Stokes' solution for a sphere in a viscous flow, using $\mathrm{C}$ as the hydrodynamic radius. The results found seem to support the proposal of Westbrook (2008). However, due to the data dispersion, it is not possible to make a more detailed analysis.

Figure 7 shows the $\mathrm{Be}$ and Re numbers, using $\mathrm{C}$ as characteristic length, for the hexagonal ice crystals (black circles) measured on this study and for the columnar ice crystals (open circles) reported by Bürgesser et al. (2016). Both data sets show an excellent agreement and this seems to indicate that an unique Be-Re relationship can be used for both crystalline habits. The best Be-Re power-law relationship using the data for hexagonal and columnar ice crystals, for Re number between 0.1 and 1 , has the same form as that of equation 3 , but in this case $A=(66 \pm 6)$ and $\beta=(1.46 \pm 0.03)$ with a $R^{2}$ value of 0.86161 .

Figure 8 shows the angle $\alpha$ between $\mathrm{D}$ and the vertical direction and the angle $\beta$ between the larger dimension (D) and the shorter dimension (d) versus Reynolds number. No trend can be observed between these angles and Re suggesting a random orientation of the ice crystals during free fall. The random orientation is in agreement with the results reported by Sassen (1980). This researcher found that planar ice crystals 
will fall with horizontal orientation for Reynolds number larger than 1, in agreement with Willmarth et al. (1964). However, Bréon and Dubruelle (2004) found oriented plates for Reynolds number between 0.3 and 80 using a simple aerodynamic model. Foster and Hallett (2002) visually confirmed that ice crystals show a random orientation after seeding and a tendency to horizontal alignment as the ice crystals grow.

\section{Conclusion}

The terminal fall velocity, size and orientation of plate-like ice crystals have been measured. The ice crystals measured present sizes between 50 and $250 \mu \mathrm{m}$ and do not show a dependence of the terminal velocity on temperature for the temperatures used on the experimental measurements.

The data collected are in agreement with the data reported by Michelli (1977) but show values lower than those reported by Kajikawa (1973). However, there is evidence that Kajikawa (1973) underestimated ice crystal size, at least for the smaller ice crystals measured, which supports the results of the present study.

No better representation of the fall velocity was obtained using the capacitance as proposed by Westbrook et al. (2008). Despite the data dispersion observed, a simple linear relationship of the terminal velocity with size and with capacitance of the ice crystals was found. Given that the size of ice crystals is the main parameter measured using remote sensing systems, a simple relationship between the fall velocity and ice crystal size could be useful.

Random orientation of the falling ice crystal was experimentally observed, which is in agreement with the theoretical model for Reynolds number less than 1.

An empirical Be-Re power-law was obtained for Reynolds number between 0.1 and 1 for hexagonal planar ice crystals. This power-law is 


$$
B e=42 R e^{0.84}
$$

This power-law is similar to the Be-Re relationship for spheres of radius $\mathrm{C}$ in Stokes flow. However, the large dispersion observed on the experimental data, which seems to be due to the variation on the ice crystal mass for ice crystals with the same size, does not allow further analysis but indicates the need for a better ice crystal mass parameterization.

The data for hexagonal and columnar ice crystals were compared and the fall velocity of columnar ice crystals show a larger velocity that hexagonal ice crystals with the same value of C. However, both crystalline habits show an unique empirical Be-Re relationship, which is

$$
B e=66 R e^{1.46}
$$

This empirical power-law is valid for Re number between 0.01 and 1 , and for hexagonal and columnar ice crystals.

\section{Acknowledgments}

This work was supported by the Secretaría de Ciencia y Tecnología de la Universidad Nacional de Córdoba (05/B493).

\section{References}

Auer Jr AH, Veal, DL. 1970. The dimension of ice crystals in natural clouds. Journal of the atmospheric sciences, 27(6), 919-926. 
Bailey MP, Hallett J. 2009. A comprehensive habit diagram for atmospheric ice crystals: Confirmation from the laboratory, AIRS II, and other field studies. Journal of the Atmospheric Sciences, 66(9), 2888-2899.

Bréon FM, Dubrulle B. 2004. Horizontally oriented plates in clouds. Journal of the atmospheric sciences, 61(23), 2888-2898.

Bürgesser RE, Ávila EE, Castellano NE. 2016. Laboratory measurements of sedimentation velocity of columnar ice crystals. Quarterly Journal of the Royal Meteorological Society, 142(697), 1713-1720.

Castellano NE, Ávila EE, Bürgesser RE, Saunders CP. 2014. The growth of ice particles in a mixed phase environment based on laboratory observations. Atmospheric Research, 150, 12-20.

Davis CI. 1974. The ice-nucleating characteristics of various AgI aerosols, Ph. D. Dissertation, University of Wyoming, $267 \mathrm{pp}$.

Foster TC, Hallett J. 2002. The alignment of ice crystals in changing electric fields. Atmospheric research, 62(1), 149-169.

Heymsfield AJ. 1972. Ice crystal terminal velocities. Journal of the Atmospheric Sciences, 29(7), 1348-1357.

Heymsfield AJ, Kajikawa M. 1987. An improved approach to calculating terminal velocities of plate-like crystals and graupel. Journal of the atmospheric sciences, 44(7), 1088-1099.

Heymsfield AJ, Westbrook CD. 2010. Advances in the estimation of ice particle fall speeds using laboratory and field measurements. Journal of the Atmospheric Sciences, 67(8), 2469-2482.

Jayaweera KOLF, Ryan BF. 1972. Terminal velocities of ice crystals. Q. J. R. Meteorol. Soc. 98: 193-197 
Kajikawa M. 1973. Laboratory measurements of falling velocity of individual ice crystals. J. Meteor. Soc. Japan, 51, 263-271.

Kajikawa M. 1975. Measurement of falling velocity of individual graupel particles. Journal of the Meteorological Society of Japan. Ser. II, 53(6), 476-481.

Khvorostyanov VI, Curry JA. 2002. Terminal velocities of droplets and crystals: Power laws with continuous parameters over the size spectrum. Journal of the atmospheric sciences, 59(11), 1872-1884.

Khvorostyanov VI, Curry JA. 2005. The theory of ice nucleation by heterogeneous freezing of deliquescent mixed CCN. Part II: Parcel model simulation. Journal of the atmospheric sciences, 62(2), 261-285.

Michaeli G. 1977. Settling velocities of small ice crystals. Tellus, 29(3), 282285.

Mitchell DL. 1996. Use of mass-and area-dimensional power laws for determining precipitation particle terminal velocities. Journal of the atmospheric sciences, 53(12), 1710-1723.

Mitchell DL, Heymsfield AJ. 2005. Refinements in the treatment of ice particle terminal velocities, highlighting aggregates. Journal of the atmospheric sciences, 62(5), $1637-1644$.

Ono A. 1970. Growth mode of ice crystals in natural clouds. J. Atmos. Sci. 27: 649-658.

Ryan B F, Wishart ER, Shaw DE. 1976. The growth rates and densities of ice crystals between-3 C and-21 C. Journal of the Atmospheric Sciences, 33(5), 842-850.

Sassen K. 1980. Remote sensing of planar ice crystal fall attitudes. Journal of the Meteorological Society of Japan. Ser. II, 58(5), 422-429. 
Schmitt C G, Heymsfield A J. 2009. The size distribution and mass-weighted terminal velocity of low-latitude tropopause cirrus crystal populations. Journal of the Atmospheric Sciences, 66(7), 2013-2028.

Um J, McFarquhar GM, Hong YP, Lee SS, Jung CH, Lawson RP, Mo Q. 2015. Dimensions and aspect ratios of natural ice crystals. Atmos. Chem. Phys. 15:3933-3956. Westbrook CD, Hogan RJ, Illingworth AJ. 2008. The capacitance of pristine ice crystals and aggregate snowflakes. arXiv preprint physics $/ 0610038$.

Willmarth WW, Hawk NE, Harvey RL. 1964. Steady and unsteady motions and wakes of freely falling disks. The Physics of Fluids, 7(2), 197-208.

\section{Captions}

Figure 1. Experimental device. Adapted from Bürgesser et al. (2016).

Figure 2. (Left panel) Real size (D and L) of an hexagonal plate-like ice crystal and (Right panel) projection of an hexagonal plate-like ice crystal over a plane. D, $d$ and $\alpha$ are the parameters measured.

Figure 3. Size distribution of the hexagonal plate-like ice crystals as box charts for $\mathrm{T}=-13,-16$ and $-20{ }^{\circ} \mathrm{C}$. The squares and horizontal bars inside the boxes respectively indicate the mean and median for ice crystal diameters. The boxes indicate the standard deviation, and the small dashes indicate the minimum and maximum ice crystal diameters. 
Figure 4. Fall velocity of plate-like ice crystals as a function of D for temperatures: $13^{\circ} \mathrm{C}$ (black circles), $-16^{\circ} \mathrm{C}$ (black triangles) and $-20^{\circ} \mathrm{C}$ (black stars) and the data reported by Kajikawa (1973) (open squares) and Michaeli (1977) (open triangles).

Figure 5. Fall velocity of plate-like ice crystals as a function of D (Upper panel) and as a function of $\mathrm{C}$ (Lower panel). Open circles represent ice crystals with horizontal orientation $\left(\alpha=90^{\circ}\right)$ and black circles represent ice crystal with random falling orientation $\left(\alpha \neq 90^{\circ}\right)$ except for the horizontal one. The solid lines represent the best linear fit for the collected data. The broken line, on the lower panel, represents the linear fit reported by Bürgesser et al. (2016) for columnar ice crystals.

Figure 6. Best number as a function of Reynolds number for the measured data for horizontal orientation (open circles) and random orientation (black circles), for the mean data reported by Kajikawa (1973) (open squares) and for the data reported by Michaeli (1977) (open triangles). The error on the Best number represents the mass variability. Solid line represents the empirical power-law found.

Figure 7. Best number as a function of Reynolds number for hexagonal ice crystals (black circles) and for columnar ice crystals (open circles) reported by Bürgesser et al. (2016).

Figure 8. The angle $\alpha$ between $\mathrm{D}$ and the vertical direction (black circles) and the angle $\beta$ between the larger dimension (D) and the shorter dimension (d) (open circles) versus Reynolds number. 


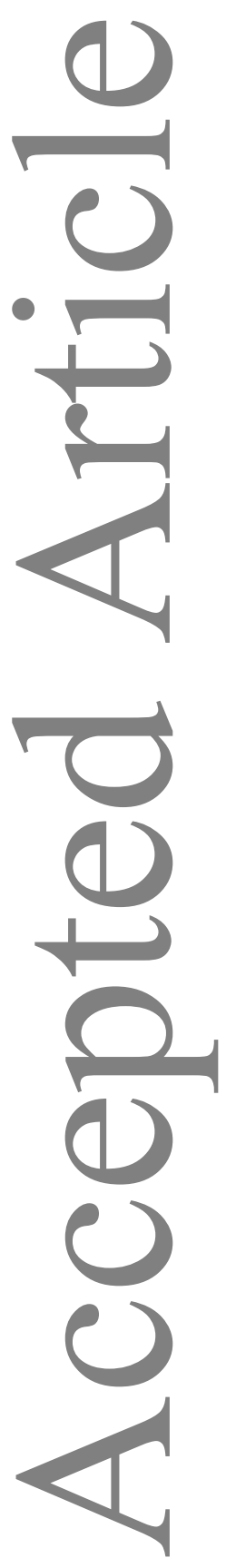

This article is protected by copyright. All rights reserved. 


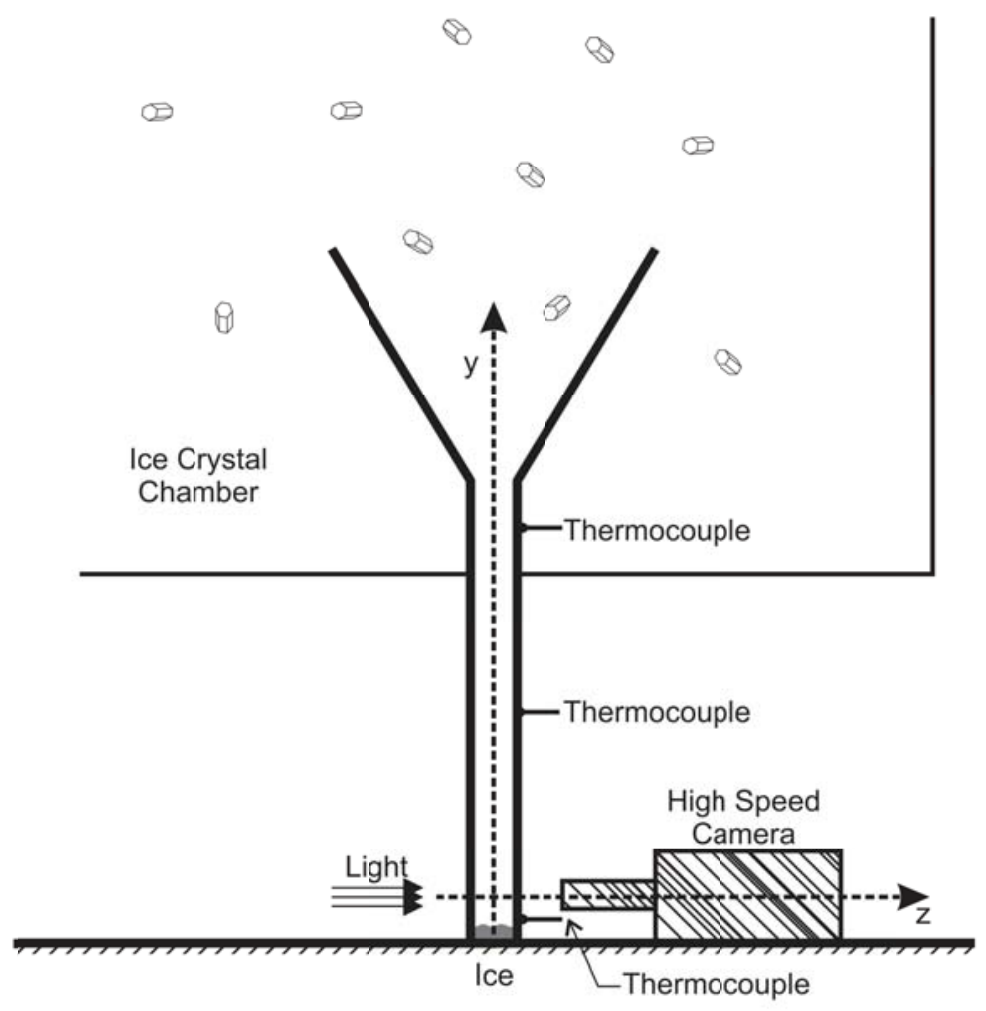



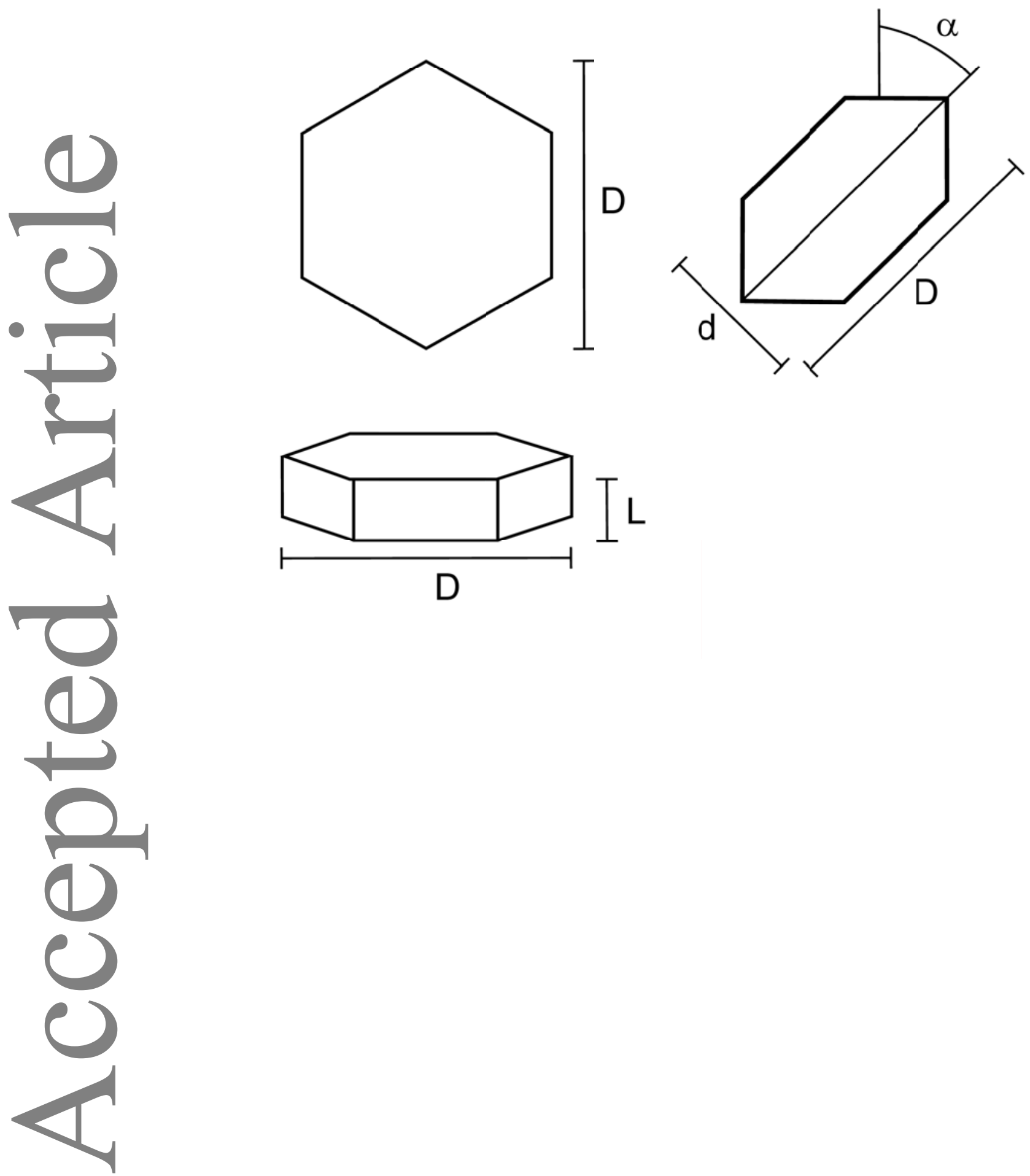

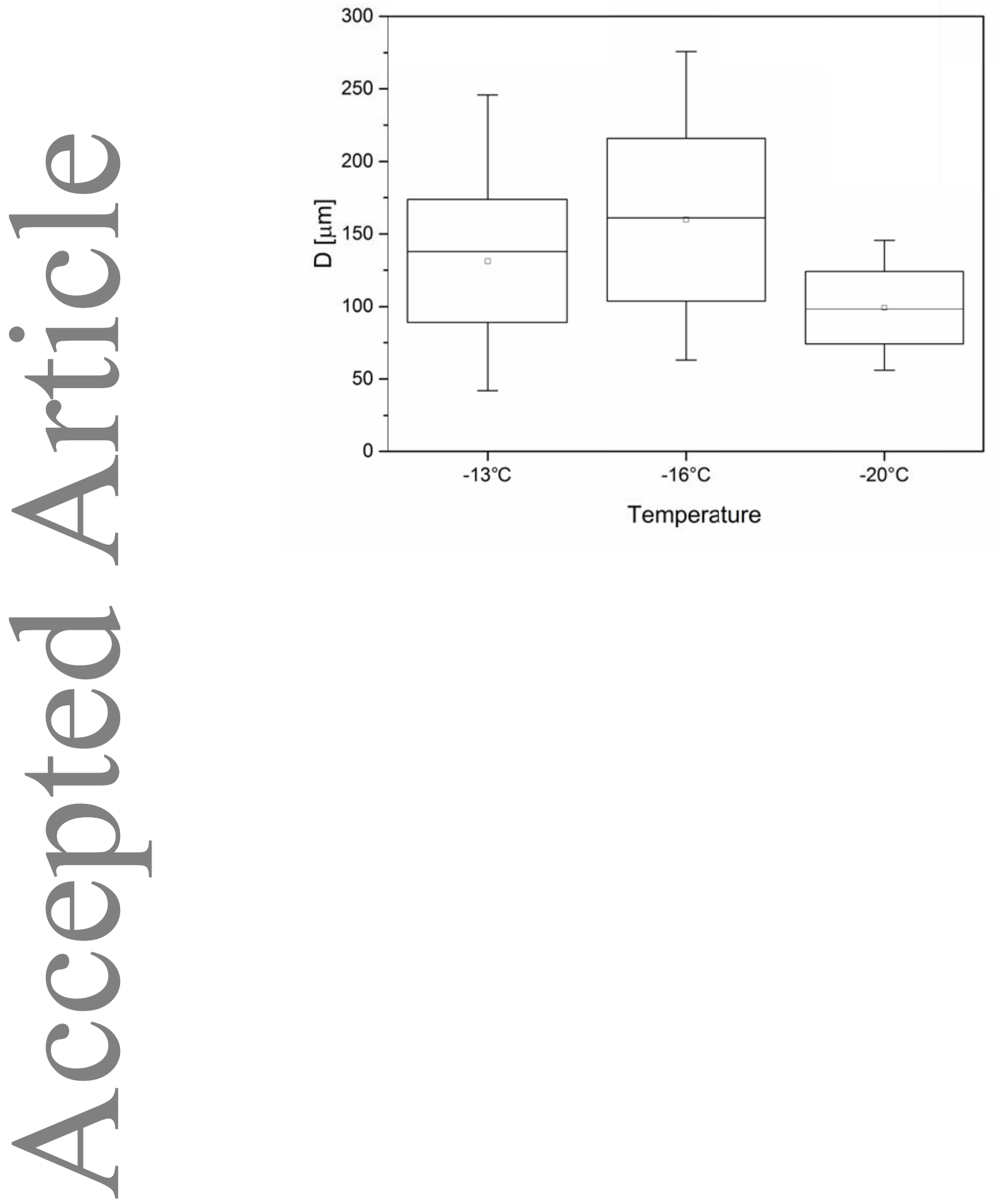

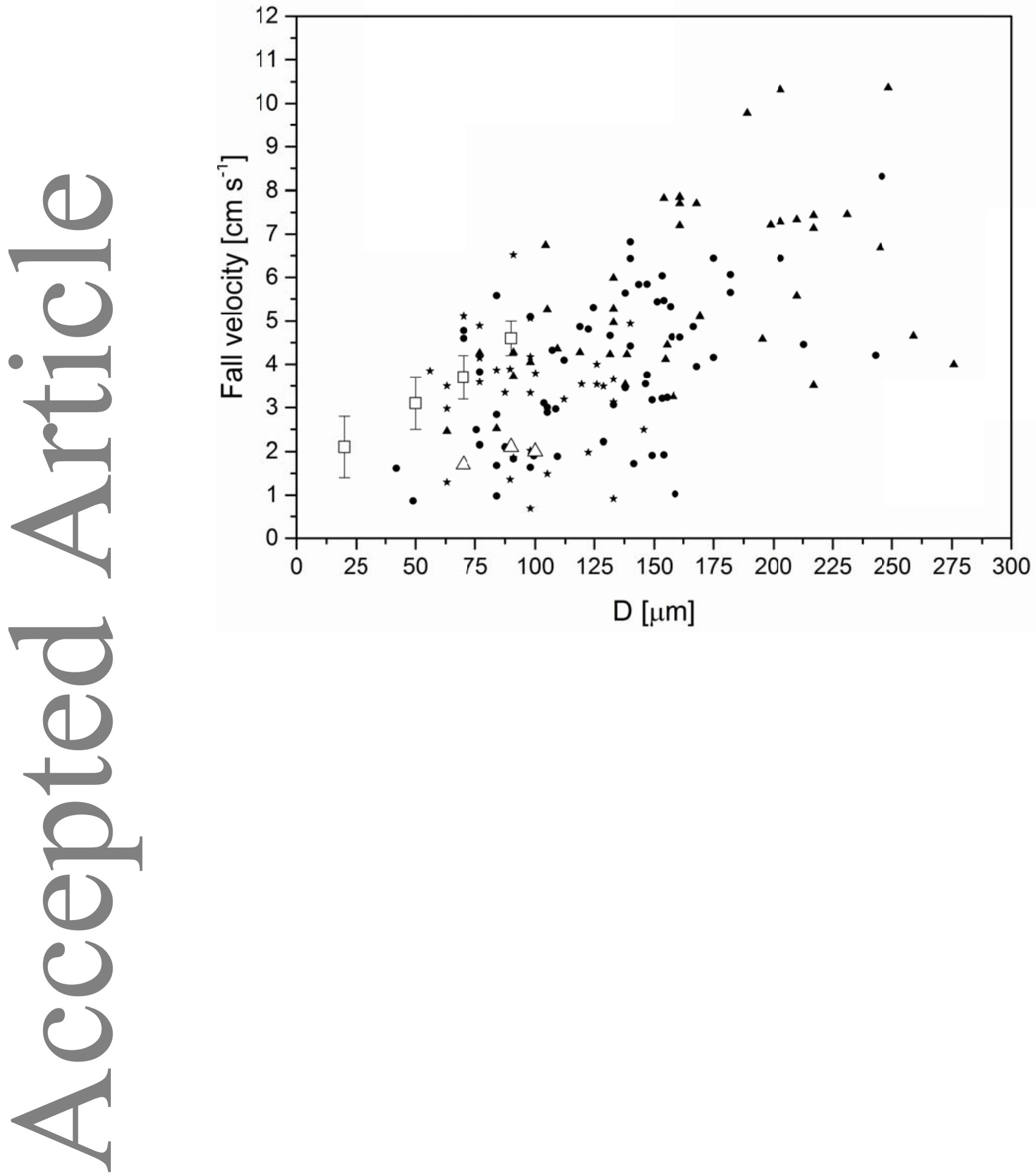

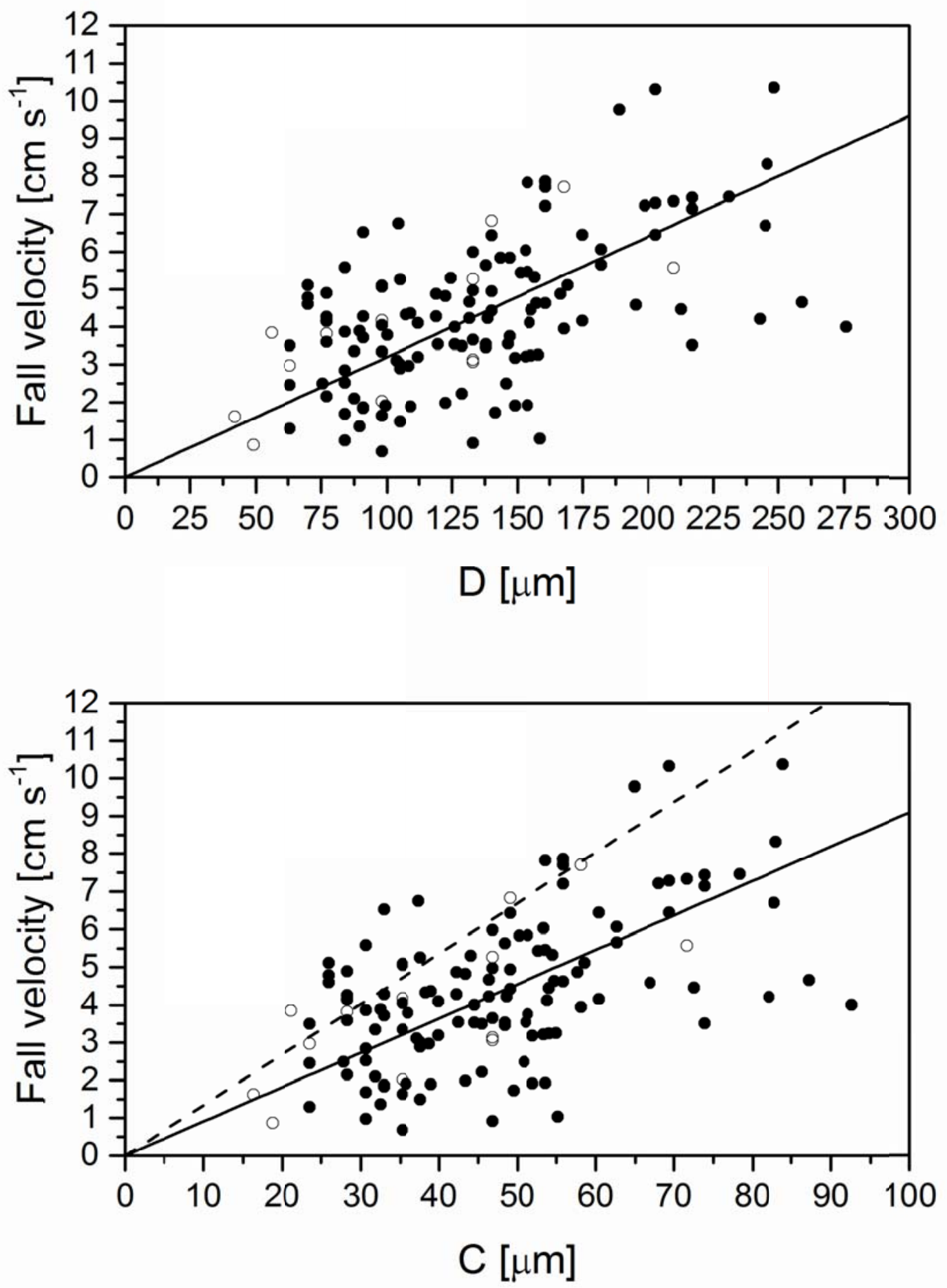

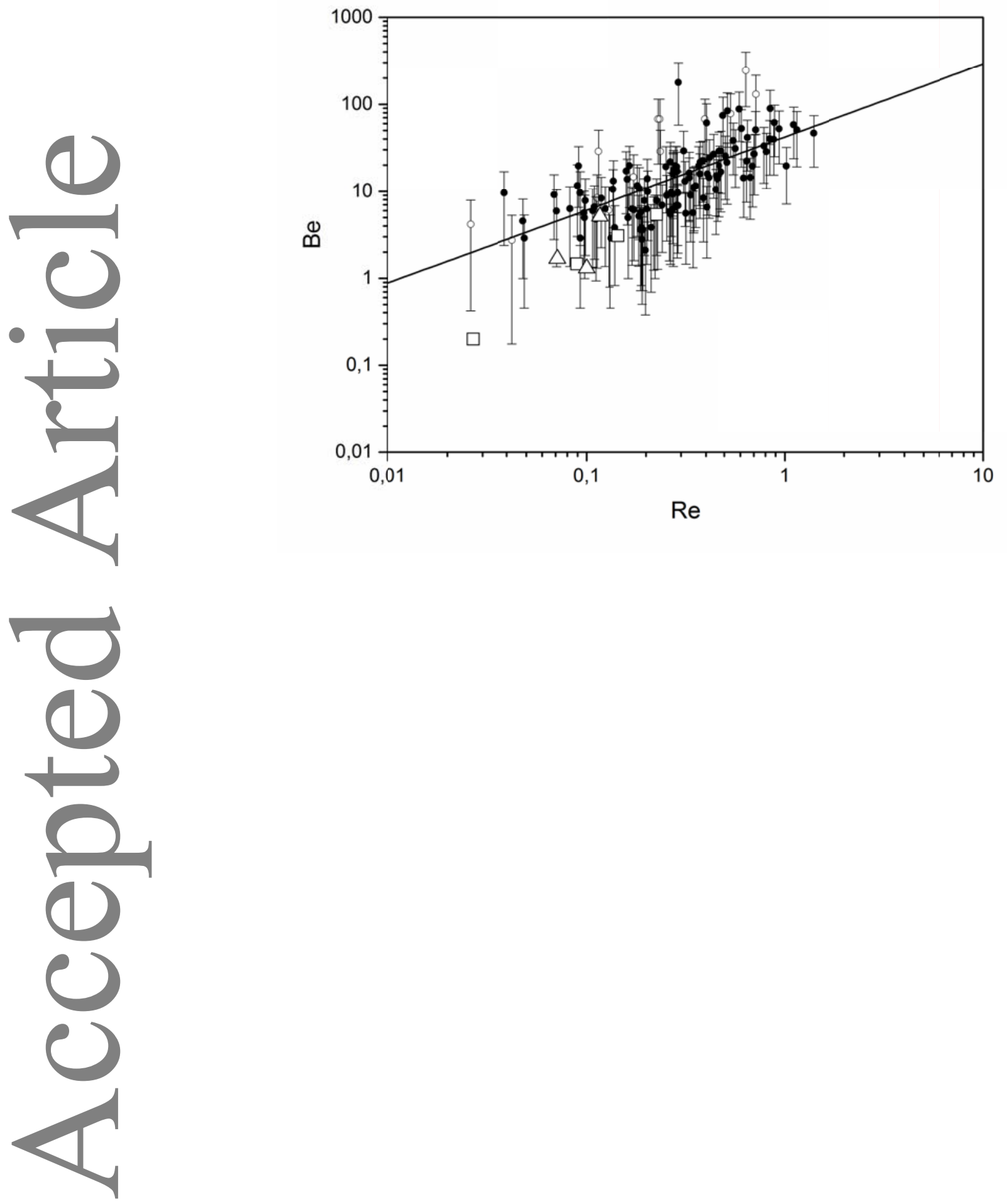

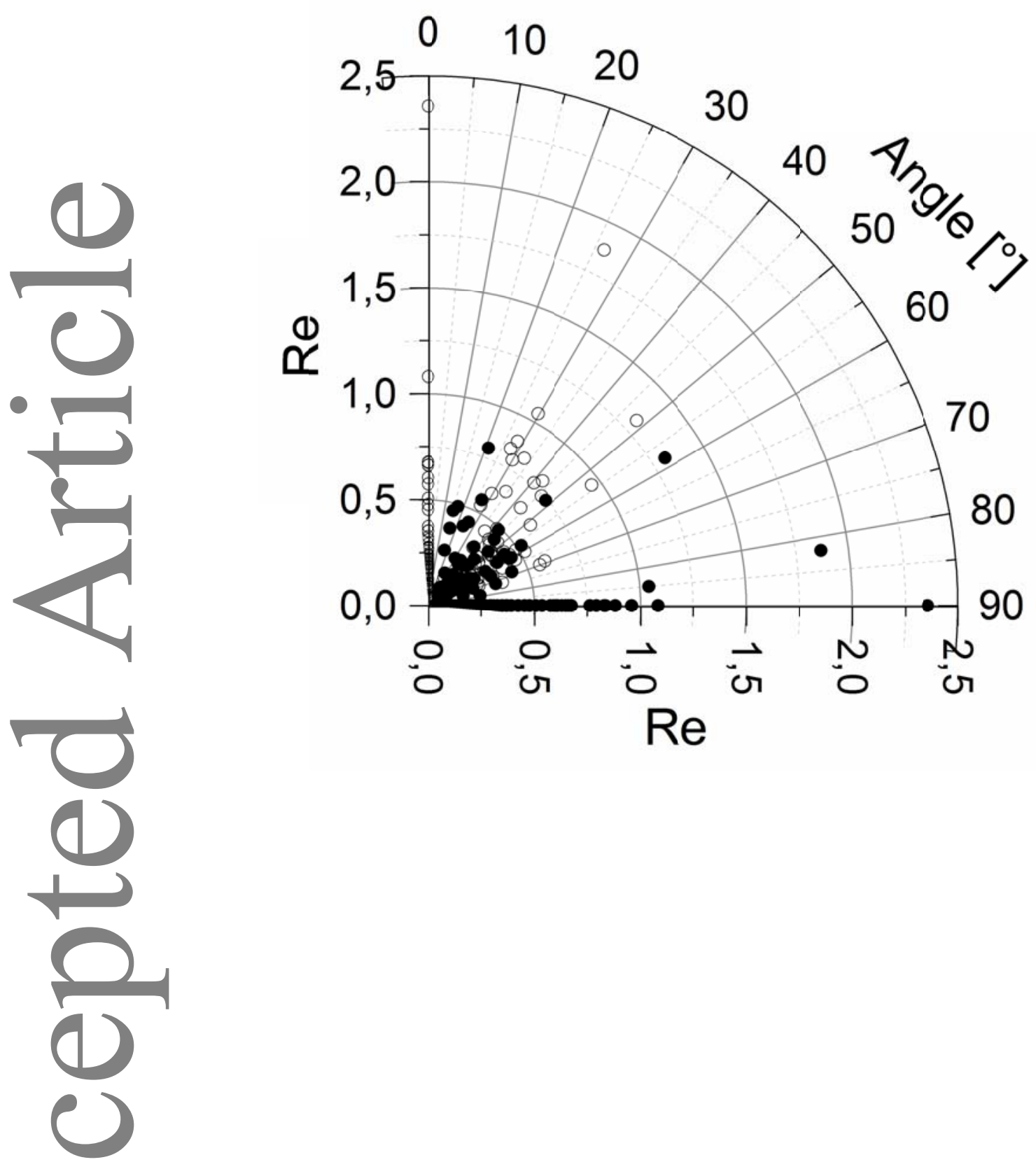

P

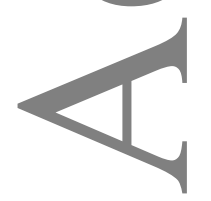

